\title{
NEW METHODS OF DETERMINING SPACECRAFT ATTITUDE
}

\author{
R. PITTS \\ Staff Member of the IUE Observatory/NASA and Astronomy Programs, \\ Computer Sciences Corporation, 10000A Aerospace Road, Lanham-Seabrook, MD 20706
}

T. JACKSON

Staff Member of the IUE Observatory/NASA and Bendix Field Engineering Corporation, One Bendix Road, Columbia MD 21045

and

R. GILMOZZI

Former Staff Member of the IUE Observatory/ESA

and ESA/Space Telescope Science Institute,

3700 San Martin Drive, Baltimore, MD 21218

\begin{abstract}
The IUE spacecraft was launched with prime and redundant mechanical Panoramic Attitude Sensors (PAS) to determine coarse spacecraft pointing. Attitude determination typically took at least 24 hours. After launch both systems failed. A new method was developed which required pointing the spacecraft at the antisolar position. After the failure of the 4th IUE gyro, it was no longer possible to point in the antisolar direction. A second method was developed which utilizes IUE's ability to track the sun with a solid state two-dimensional sun sensor. Attitude determination can now be completed in several hours. An hour is required for coarse position measurement and several more hours are needed, using a small 15 arc minute square finder camera, for final attitude confirmation. These methods should be of use for other spacecraft where weight is critical or there is a desire to avoid mechanical devices.
\end{abstract}

\section{Introduction}

The International Ultraviolet Explorer (IUE) is a geosynchronous orbiting telescope launched by the National Aeronautics and Space Administration (NASA) on January 26, 1978, and operated jointly by NASA and the European Space Agency. A description of the spacecraft and science instrument is given by Boggess et al. (1978a, b). The science instrument consists of two spectrographs which span the wavelength range of 1150 to $3200 \AA$ and offer two dispersions with resolutions of $7 \AA$ and $0.2 \AA$. A discussion of the science instrument characteristics and the processing of the telescope raw data is given by Turnrose et al. $(1981,1984)$. A description of the spacecraft's mechanical and electronic systems is found in the System Design Report (1976a, b). A general history of the spacecraft has been written by Boggess et al. (1987) and a description of the Observatory operations given by Fälker et al. (1987). During the twelve years that the spacecraft has been on station, 4 of the 6 gyroscopes, which were originally designed to provide the attitude reference required for pointing and slewing of the telescope, have failed. Since the failure of the 4th gyro, an attitude control system has been employed which utilizes a solid state, two-dimensional Fine Sun Sensor (FSS) to provide the third axis of stabilization.

Y. Kondo (ed.), Observatories in Earth Orbit and Beyond, 521-524.

(C) 1990 Kluwer Academic Publishers. Printed in The Netherlands. 
A discussion of this system is given by Femiano (1986). A third control system, which requires a single gyro and uses the second dimension of the FSS to maintain three-axis stabilization, has been developed and will be employed upon the failure of the 5th gyro.

\section{Spacecraft Attitude Control}

The IUE spacecraft can move about any one of three independent axes. The terminology of attitude control has been adapted from aviation. If an airplane is on course in level flight, an up/down motion of the nose of the craft is called "pitch", a movement of the plane from side to side is called "yaw", and a turning about the axis of the fuselage is called "roll". Analogously, the IUE can be considered "on course", when the telescope is pointing directly away from the sun. Pitch is toward/away from the sun, yaw is a great circle perpendicular to the pitch great circle, and roll is motion about the telescope tube axis. The north celestial pole projected onto the spacecraft roll plane provides a reference point for roll. The system is local as it moves with the spacecraft, but since it is defined with w.r.t the sun and north celestial pole, transformations using Euler angles can be made from the more familiar coordinates of right ascension (RA) and declination (DEC) to that of the spacecraft's pitch, yaw, and roll. For discussions of spacecraft attitude determination and control, see Wertz (1987).

Until recently, earthbased telescopes were built with one axis of the telescope aligned with the Earth's axis of rotation. Such telescopes can be moved in the declination axis and set to a particular value. Motion about the polar axis allows for setting the RA value and tracking a star as the Earth rotates. With the IUE, the Beta angle (i.e. a measurement of the pitch angle) can be set. The telescope is then rotated simultaneously in yaw and roll so that the Beta angle remains constant until the pointing position of the target is reached (i.e. the spacecraft attitude). One complication is that the sun, as seen by telescope, changes postion as the IUE orbits the sun. Thus emphemeride calculations are part of every maneuver calculation for the spacecraft.

\section{Attitude Determination}

Infrequently, an error condition in the spacecraft onboard computer or the ground system will result in a loss of spacecraft attitude. To recover attitude, coarse pointing must first be determined (i.e. the current position to within several degrees of arc). Next a Fine Error Sensor (FES) with a field of view of 15.8 arc minutes square is used to determine exact pointing. At launch, a Panoramic Attitude Sensor (PAS) was used to determine coarse attitude. The technique relied on the positions of sun and Earth seen by the IUE to accomplish coarse attitude determination and required 24 hours to complete.

A second method of attitude determination was developed after launch which took much less time and which did not rely on the PAS, both of which have since failed. This method, conceptualized by A. Holm, and developed by A. Holm and F. Schiffer (Holm, 1990), makes use of the relationship between the local spacecraft 
axes and RA and DEC. If the spacecraft is pointed in the antisolar direction, its Beta and yaw angles are zero. The RA and DEC of the antisolar position can be accurately calculated for any given time. An FES image can then be taken and the field matched to a Palomar Sky Survey (POSS) chart. From the match, the current spacecraft roll at that position (i.e. w.r.t. the north celestial pole) can be estimated. Assuming this roll, the spacecraft is maneuvered to a target a few degrees away. After locating the target with a second FES image, the process is repeated to obtain a refined estimate of the spacecraft roll. As a final check, the telescope is maneuvered to a target at a Beta angle of 15 to 20 degrees of arc before resuming normal slewing. The process takes two to three hours. Since the initial attitude determination is made at a Beta angle of zero, it was named the Betazero method.

After adoption of the two-gyro/FSS control system, the Betazero method could no longer be used since the FSS heads lose sun presence below a Beta angle of 15 degrees. A new method was conceptualized by $\mathrm{R}$. Pitts and C. Imhoff, and developed by R. Gilmozzi. The method utilizes the solar motion as seen by the IUE. The telescope is pointed toward a star in the current FES field of view. The spacecraft is then locked to the solar motion in pitch by using the FSS sensor. As the spacecraft tracks the sun, the star slowly moves across the FES field of view while the magnitude and sign of its motion in pitch are measured. Again using the relationship between pitch, yaw, and roll, and RA and DEC, two possible solutions of the position of the spacecraft are obtained. The sign of the roll, determined from other spacecraft telemetry, allows the selection of the correct solution. This provides the coarse attitude determination to within 1 to 3 degrees with all uncertainty being in the yaw axis. Several FES fields are then taken as the spacecraft position is changed by small amounts in yaw. These are then matched to the POSS charts of the area to complete the attitude recovery. The method requires several hours to perform. Since it is based on measuring the change in Beta angle of a target due to solar motion, it was named the Betadot method. A complete description is given by Gilmozzi et al.. (1990). Recently, T. Jackson has written an on-line computer program based on an equivalent mathematical derivation using conic sections which employs two computed measurements of the antisolar position rather than directly measuring the rate of change of solar position. Again, the sign of the roll angle is determined by other spacecraft telemetry. He has also modified the Betadot command sequence sent to the spacecraft which has improved the $\mathrm{S} / \mathrm{N}$ of the telemetry measurements so that a full attitude recovery can now be completed in from one to two hours.

\section{Acknowledgements}

This work is supported by NASA contracts NAS 5-29375 and NAS 5-31000. We gratefully acknowledge the sigificant contributions of the designers, engineers, and software specialists at GSFC and OAO which maintain the high productivity of the aging IUE spacecraft which recently celebrated its twelfth anniversary on station. 


\section{References}

Boggess, A., and Wilson, R.: 1987, in The History of IUE, ed(s)., 3, Exploring the Universe with the IUE Satellite, ed. Y. Kondo, D. Reidel, Dordrecht

Boggess, A., Carr, F., Evans, D., et al..: 1978a, Nature 275, 372-377

Boggess, A., Bohlin, R., Evans, D., et al.: 1978b, Nature 275, 377-385

Fälker, J., Gordon, F., and Sandford, M.: 1987, in Operation of a Multi-Year, Multi-Agency Project, ed(s)., 21, Exploring the Universe with the IUE Satellite, ed. Y. Kondo, D. Reidel, Dordrecht

Femiano, M.: 1986, in Inflight Redesign of the IUE Attitude Control System, ed(s)., 164, AIAA Space Systems Technology Conference, American Institute of Aeronautics and Astronautics, AIAA-86-1193-CP

Gilmozzi, R., and Pitts, R.: 1990, submitted to AIAA Journal of Spacecraft and Rockets

Holm, A.: 1990, private communication

Wertz, J. (ed.): 1978, Spacecraft Attitude Determination and Control, D. Reidel, Dordrecht

System Design Report: 1976a, System Design Report of IUE, Vol. I, NASA/GSFC IUE401-76-099

System Design Report: 1976b, System Design Report of IUE, Vol. II, NASA/GSFC IUE401-76-099

Turnrose, B., and Thompson, R.: 1984, International Ultraviolet Explorer Image Processing Information Manual, Version 2.0, Computer Sciences Corporation, CSC/TM-84/6058 under NASA Contract NAS 5-27295

Turnrose, B., Harvel, C., and Stone, D.: 1981, International Ultraviolet Explorer Image Processing Information Manual, Version 1.1, Computer Sciences Corporation, CSC/TM-81/6268 under NASA Contract NAS 5-24350 